\title{
In memoriam Friedrich Roth (1931-2018)
}

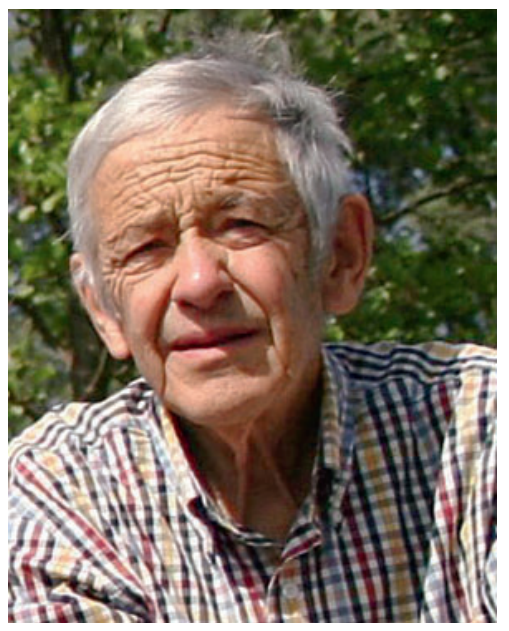

Friedrich Roth

\section{Ein Pionier der Intensivmedizin}

Am 13. April 2018 hat die Schweiz mit Prof. Friedrich Roth, einen Tag nach dessen 87. Geburtstag, eine herausragende Persönlichkeit verloren, die ihr berufliches Wirken der Behandlung von schwerstkranken und schwerstverletzten Patientinnen und Patienten verschrieben hatte.

Schon bald war Dr. Friedrich Roth ein gewichtiges Mitglied des behandelnden Teams.

Nachdem im Kommunehospitalet in Kopenhagen in der Zeit der Polioepidemie 1953 die erste «Intensivstation" gegründet worden war, begann die Intensivbehandlung in Bern in den Jahren 1956/57 mit der Behandlung von Tetanus-Patienten. Schon bald war Dr. Friedrich Roth ein gewichtiges Mitglied des behandelnden Teams. In der Folge wurde er im Auftrag von Prof. B. Tschirren, dem Leiter der damaligen zentralen Anästhesieabteilung des Inselspitals, mit dem weiteren Ausbau beauftragt und 1967 zum Leiter der «Abteilung für Reanimation und Intensivbehandlung» befördert. Diese Entwicklung war auch dem damaligen Chefarzt der Chirurgischen Klinik, Prof. K. Lenggen- hager, zu verdanken - hatte dieser doch schon früh erkannt, dass der Fachbereich Anästhesie der ideale Partner für die perioperative und unmittelbar postoperative Betreuung der Patientinnen und Patienten war. Damit war ein wichtiger Grundstein gelegt zum künftigen Weg einer selbständigen, interdisziplinären Entwicklung des neuen Fachgebietes der Intensivmedizin.

1967 wurde er zum Leiter der «Abteilung für Reanimation und Intensivbehandlung" befördert.

Friedrich Roth besuchte das Gymnasium in Burgdorf. Er schloss das Medizinstudium 1956 in Bern ab und erhielt 1958 den Doktortitel aufgrund der Dissertation «Zur Wirkungsweise der venösen Luftembolie». 19571961 wirkte er als Assistenzarzt an verschiedenen Kliniken, darunter am renommierten Alder Hey Children's Hospital in Liverpool, eine Erfahrung, von welcher er viele Jahre lang zehrte. 1962 wurde Prof. Friedrich Roth an der Anästhesieabteilung von Prof. B. Tschirren zum Oberarzt, 1964 zum Chefarzt-Stellvertreter befördert.

\section{Gründung der Berner Schule für Weiterbildung in Intensivpflege}

Der Aufbau der Intensivstation war ein schwieriges Unterfangen, gab es doch noch kein entsprechend ausgebildetes Personal. Friedrich Roth gründete deshalb die Schule für die Weiterbildung in Intensivpflege und führte sie zu ihrem schweizweit hervorragenden Ruf als Weiterbildungsstätte für Pflegende. Bis zu seiner Pensionierung 1995 wurden unter seiner Leitung hunderte von spezialisierten Intensivpflegefachleuten ausgebildet. Aber auch zahllose Ärztinnen und Ärzte haben von seiner auf Praxis, steter Innovation, Sicherheit und Menschlichkeit orientierten Ausbildung profitiert. Einer stattlichen Anzahl wurde in der Folge die ärztliche respektive pflegerische Leitung in anderen Intensivstationen der Schweiz anvertraut.

Friedrich Roth war 1972 Gründungsmitglied der Schweizerischen Gesellschaft für Intensivmedizin 
(SGI-SSMI). Auch in dieser Fachgesellschaft hat er sich für Aufbau und Weiterentwicklung der Intensivmedizin und speziell auch für die Weiterbildung der Pflegenden eingesetzt. Wichtiges Zwischenziel war diesbezüglich die Schaffung des «Fähigkeitsausweises für Intensivpflege» für Pflegefachpersonen. In Würdigung seiner Verdienste um die interprofessionell gelebte Intensivmedizin erhielt er 2006 die Ehrenmitgliedschaft der SGI-SSMI.

\section{Erstes Unispital mit einer Klinik für interdisziplinäre Intensivmedizin}

Gegen Ende seiner Amtszeit schuf Prof. Friedrich Roth die Voraussetzungen dafür, dass alle Intensivstationen des Inselspitals zu einer eigenständigen Klinik für Intensivmedizin zusammengeführt werden konnten. Er wurde darin von seinem damaligen Vorgesetzten, Prof. D. Thomson, unterstützt, welcher bereit war, die Intensivstation aus dem Institut für Anästhesiologie und Intensivmedizin herauszulösen und ihr einen eigenständigen Status zu geben. Damit wurde das Inselspital das erste Universitätsspital der Schweiz mit einer Klinik für interdisziplinäre Intensivmedizin.

\section{Korrespondenz:}

Prof. Dr. med.

Hans Ulrich Rothen

Rabbentalstr. 65

CH-3013 Bern

Hu_rothen[at]bluewin.ch

Von der Medizinischen Fakultät der Universität Bern wurde er in Anerkennung seiner Verdienste für Lehre und Forschung mit dem Professorentitel geehrt. Seine Experimente und wissenschaftlichen Untersuchungen hat er im stillen Kämmerlein durchgeführt; zur Publikation fehlte ihm die Zeit. Was er in der Klinik anwen-

Viele seiner Patientinnen und Patienten haben dank seiner von ihm entwickelten Methoden und seinem persönlichen, selbstlosen Engagement überlebt.

dete, probierte er - gelegentlich sehr abenteuerlich zuerst an sich selber aus, und viele seiner Innovationen sind heute in der Intensivmedizin Standard.

Prof. Friedrich Roth hatte für alle eine offene Tür, ein offenes Ohr. Jedem begegnete er unvoreingenommen und mit Achtung, und sein Name war ein Türöffner, weil er für Anstand, Engagement und Lauterkeit bürgte. Viele seiner Patientinnen und Patienten haben dank seiner von ihm entwickelten Methoden und seinem persönlichen, selbstlosen Engagement überlebt. Prof. Friedrich Roth hat medizinisch, moralisch und menschlich unsere Leben geprägt; es war ein Privileg, mit ihm zusammenarbeiten zu dürfen.

Im Namen seiner ehemaligen Mitarbeiterinnen und Mitarbeiter

Bruno Regli, Thun

Hans Ulrich Rothen, Bern
Schmid, Bremgarten b. Bern 